\title{
Functional imaging for pheochromocytoma-paraganglioma: a step closer to understanding its place in clinical practice
}

\author{
Camilo Jimenez $^{1} \cdot$ Steven G. Waguespack $^{1}$
}

Received: 23 April 2015/ Accepted: 19 June 2015/Published online: 26 June 2015

(C) Springer Science+Business Media New York 2015

Pheochromocytomas-paragangliomas (PPG) are rare tumors that, when originating in the adrenal medulla or sympathetic paraganglia, are characterized by increased synthesis and secretion of catecholamines and expression of catecholamine reuptake transporters. Furthermore, as neuroendocrine tumors, PPG may express cell membrane somatostatin receptors. These characteristics make PPG amenable to various functional imaging (FI) modalities that, in conjunction with anatomic imaging (AI) such as computed tomography (CT) and magnetic resonance imaging (MRI), can assist with the localization and characterization of these unique neoplasms. Several FI modalities are currently available, including (i) target catecholamine physiology $\left[{ }^{123} \mathrm{I}\right.$ - or ${ }^{131}$ I-meta-iodobenzylguanidine (MIBG); ${ }^{18} \mathrm{~F}$-fluoro-dihydroxyphenylalanine $\left({ }^{18} \mathrm{~F}\right.$-DOPA) positron emission tomography (PET); ${ }^{18} \mathrm{~F}$ fluorodopamine $\left({ }^{18} \mathrm{~F}\right.$-FDA) PET], (ii) identify somatostatin receptor expression [ ${ }^{111}$ In-DTPA-pentetreotide (octreotide); ${ }^{68} \mathrm{Ga}$-DOTATATE PET], or (iii) target increased tumoral glucose uptake $\left[{ }^{18} \mathrm{~F}\right.$-fluorodeoxyglucose (FDG) PET].

The role of FI in the routine evaluation of PPG remains poorly studied, partly due to the rarity of these tumors and the fact that few centers see large numbers of such patients, which makes prospective hypothesis-driven studies difficult to achieve. Additionally, although some FI studies (such as MIBG and FDG-PET scans) are widely available for clinical use, other modalities remain experimental and

Camilo Jimenez

cjimenez@mdanderson.org

1 Department of Endocrine Neoplasia and Hormonal Disorders, The University of Texas MD Anderson Cancer Center, Houston, TX 77030, USA difficult to obtain outside of a clinical research setting. Furthermore, all FI technologies are expensive, and the cost-effectiveness of FI as part of routine clinical practice has not been studied.

The current study by Brito and colleagues [1] was commissioned by the Endocrine Society as it developed its inaugural PPG guideline [2], and the goal of the study was to describe the additive benefit of FI in PPG patients who had undergone routine AI with CT/MRI. Brito et al. [1] conducted a systematic literature review of 32 studies published from 1983 to 2012 that evaluated FI in patients with biochemically proven PPG. The systematic review included data from 1264 patients and used a clearly defined methodology. The authors found that FI testing incrementally aided in PPG localization in only 21/1445 (1.5 \%) primary tumors and 28/805 (3.5\%) of metastases, leading them to conclude that FI tests have a limited role in the routine diagnostic evaluation of PPG. In fact, in more than $95 \%$ of patients with resectable disease, CT/MRI scans were sufficient for determining appropriate treatment. Furthermore, in malignant PPG, ${ }^{18} \mathrm{~F}$-DOPA PET, and ${ }^{18} \mathrm{~F}$ FDA PET were the most successful at identifying lesions not detected via AI, providing additional benefit in $6 / 60$ $(10 \%)$ and 5/78 (6.4\%) cases, respectively.

Although a good first step in better understanding the utility of FI in the diagnosis of PPG, the current study has some unavoidable limitations. All of the studies examined lacked a control group, and most of these were retrospective in nature. Furthermore, some studies evaluated PET without simultaneous CT likely reducing the PET performance for localizing PPG. In addition, many of the primary studies did not include relevant information on the clinical and genetic aspects of PPG that have been delineated over the last decade (e.g., genotype analysis, biochemical phenotype, and clinical factors that help predict malignant 
disease). Given that the studies spanned almost three decades and that AI has significantly improved in terms of anatomic resolution over that same timespan, it is also unclear if any incremental benefit of FI would be identified in the contemporary era of AI. Finally, the current study is understandably not able to address several other unresolved clinical questions such as (i) the number of patients needed to screen for FI to be a cost-effective approach, (ii) the rate of false positive FI tests that may lead to inappropriate surgical procedures, (iii) the clinical significance (or not) of missing a small PPG or PPG metastasis that can only be identified via FI modalities, (iv) the impact of FI testing on surgical considerations such as the feasibility of and approach to surgery, (v) the utility of FI to rule out a PPG in the setting of a false positive CT/MRI, and (vi) as pointed out by the authors, the role of FI in improving patient-important outcomes.

Although the results of this meta-analysis must be interpreted cautiously, especially given the large amount of data from the primary studies that are not readily available, Brito et al. have enhanced our understanding of the role of FI in routine clinical practice. This has led the Endocrine Society to recommend FI testing only in particular clinical scenarios: in the work-up of patients at high risk for or known to have metastatic disease and in those in whom the use of therapeutic radiopharmaceuticals is a consideration.

Given our clinical experience and based upon the fact that most PPG are benign intra-abdominal tumors, we also believe that the vast majority of patients with PPG, especially those presenting with clinical signs or symptoms, will easily and accurately be diagnosed via conventional AI, a conclusion supported by the current study. However, there are still subsets of patients in whom FI may play an important role: (i) patients with clinical predictors of malignant disease [3], (ii) patients with known metastatic disease [4], especially if this may change clinical management or lead to radiopharmaceutical therapy [5], and (iii) patients with hereditary tumor syndromes, especially the familial paraganglioma syndromes, that predispose affected individuals to multifocal and extra-adrenal tumors [6].

Patients with malignant PPG are especially good candidates for FI testing. Although macroscopic lymph node, lung, and liver metastases are relatively easy to identify via conventional AI, bone metastases are not. Bone metastases, which occur in approximately $72 \%$ of malignant PPG patients, are frequently identified by FI rather than CT/MRI [7]. Therefore, knowledge of bone metastases, which may only be possible via FI, may lead the clinician to recommend local, antiresorptive, and/or systemic therapies to decrease the rate of skeletal-related events [7]. As clinical trials to treat malignant PPG are developed (www. clinicaltrials.gov), FI should be incorporated as an exploratory endpoint to correlate with objective response rates [8].

An unresolved issue in the management of patients with a germline $S D H x$ mutation is whether or not FI will play a role in the prospective screening for clinical manifestations of the familial paraganglioma syndromes. A recent study has suggested that FDG-PET/CT might be a very sensitive tool for the early identification of PPG in asymptomatic $S D H x$ carriers [9]. Further study into the efficacy and costeffectiveness of routine FI screening in this population will be required but may not be necessary given the evolution of whole body MRI as a screening modality [10]. Hypothetically, in patients with apparently sporadic tumors and a noradrenergic phenotype, FI may help identify more rapidly multifocal disease and other components of a hereditary tumor syndrome, thereby facilitating treatment planning before genetic testing is completed.

In conclusion, as demonstrated by Brito et al., the majority of PPG patients (i.e., patients with non-metastatic pheochromocytomas $<5 \mathrm{~cm}$ associated with an adrenergic phenotype) will not clearly benefit from routine FI at the time of diagnosis. In contrast, patients with clinical predictors of malignancy, known metastatic disease, or tumors associated with a noradrenergic phenotype, especially those with a known germline $S D H B$ mutation, may benefit from FI testing. The last decade has been an exciting one in the PPG field, and FI will always play an important role in the management of some of these patients. Moving forward, advances in our understanding of the pathophysiology and treatment of PPG should lead to more clarity as to how FI should be appropriately incorporated into routine clinical care in a cost-effective manner.

\section{Compliance with Ethical Standards}

Conflict of interest The authors declare that they have no conflict of interest.

\section{References}

1. J.P. Brito, N. Asi, M.R. Gionfriddo, C. Norman, A.L. Leppin, C. Zeballos-Palacios, C. Undavalli, Z. Wang, J.P. Domecq, G. Prustsky, T.A. Elraiyah, L.J. Prokop, V.M. Montori, M.H. Murad, The incremental benefit of functional imaging in pheochromocytoma/paraganglioma: a systematic review. Endocrine (2015). doi:10.1007/s12020-015-0544-7

2. J.W. Lenders, Q.Y. Duh, G. Eisenhofer, A.P. Gimenez-Roqueplo, S.K. Grebe, M.H. Murad, M. Naruse, K. Pacak, W.F. Young, E. Society, Pheochromocytoma and paraganglioma: an endocrine society clinical practice guideline. J. Clin. Endocrinol. Metab. 99(6), 1915-1942 (2014)

3. M. Ayala-Ramirez, L. Feng, M.M. Johnson, S. Ejaz, M.A. Habra, T. Rich, N. Busaidy, G.J. Cote, N. Perrier, A. Phan, S. Patel, S. Waguespack, C. Jimenez, Clinical risk factors for malignancy 
and overall survival in patients with pheochromocytomas and sympathetic paragangliomas: primary tumor size and primary tumor location as prognostic indicators. J. Clin. Endocrinol. Metab. 96(3), 717-725 (2011)

4. H.J. Timmers, A. Kozupa, C.C. Chen, J.A. Carrasquillo, A. Ling, G. Eisenhofer, K.T. Adams, D. Solis, J.W. Lenders, K. Pacak, Superiority of fluorodeoxyglucose positron emission tomography to other functional imaging techniques in the evaluation of metastatic SDHB-associated pheochromocytoma and paraganglioma. J. Clin. Oncol. 25(16), 2262-2269 (2007)

5. E. Baudin, M.A. Habra, F. Deschamps, G. Cote, F. Dumont, M. Cabanillas, J. Arfi-Roufe, A. Berdelou, B. Moon, A. Al Ghuzlan, S. Patel, S. Leboulleux, C. Jimenez, Therapy of endocrine disease: treatment of malignant pheochromocytoma and paraganglioma. Eur. J. Endocrinol. 171(3), R111-R122 (2014)

6. P.L. Dahia, Pheochromocytoma and paraganglioma pathogenesis: learning from genetic heterogeneity. Nat. Rev. Cancer 14(2), 108-119 (2014)

7. M. Ayala-Ramirez, J.L. Palmer, M.C. Hofmann, M. de la Cruz, B.S. Moon, S.G. Waguespack, M.A. Habra, C. Jimenez, Bone metastases and skeletal-related events in patients with malignant pheochromocytoma and sympathetic paraganglioma. J. Clin. Endocrinol. Metab. 98(4), 1492-1497 (2013)

8. M. Ayala-Ramirez, C.N. Chougnet, M.A. Habra, J.L. Palmer, S. Leboulleux, M.E. Cabanillas, C. Caramella, P. Anderson, A. Al Ghuzlan, S.G. Waguespack, D. Deandreis, E. Baudin, C. Jimenez, Treatment with sunitinib for patients with progressive metastatic pheochromocytomas and sympathetic paragangliomas. J. Clin. Endocrinol. Metab. 97(11), 4040-4050 (2012)

9. C. Lepoutre-Lussey, C. Caramella, F. Bidault, D. Déandreis, A. Berdelou, A. Al Ghuzlan, D. Hartl, I. Borget, A.P. GimenezRoqueplo, F. Dumont, F. Deschamps, C. Nascimento, J. Lumbroso, M. Guillaud Bataille, M. Schlumberger, E. Baudin, S. Leboulleux, Screening in asymptomatic SDHx mutation carriers: added value of ${ }^{18}$ F-FDG PET/CT at initial diagnosis and 1-year follow-up. Eur. J. Nucl. Med. Mol. Imaging 42(6), 868-876 (2015)

10. K.W. Jasperson, W. Kohlmann, A. Gammon, H. Slack, L. Buchmann, J. Hunt, A.C. Kirchhoff, H. Baskin, A. Shaaban, J.D. Schiffman, Role of rapid sequence whole-body MRI screening in SDH-associated hereditary paraganglioma families. Fam. Cancer 13(2), 257-265 (2014) 